\title{
Gestational Diabetes Mellitus a Dysfunctional Metabolic State-A Perspective
}

\section{Sheriff DS*}

Department of Andrology and Metabolism, Reprolabs, India

*Correspondence: Dhastagir Sultan Sheriff, Department of Andrology and Metabolism, Reprolabs, India

Received on 07 May 2019; Accepted on 17 June 2019; Published on 24 June 2019

Copyright (C) 2019 Sheriff DS. This is an open access article and is distributed under the Creative Commons Attribution License, which permits unrestricted use, distribution, and reproduction in any medium, provided the original work is properly cited.

\begin{abstract}
Pregnancy is considered as a test for beta cell reserve. If there is a good function, insulin resistance will overcome. If not, gestational diabetes will occur. Insulin resistance (IR) present in normal pregnancy is required to provide nutrients to the growing fetus. There is a rapid increase of insulin in such an insulin resistant state. The possibility of lipid deposition in muscle fibers (intramyocellular) could be one of the possible mechanism of IR in gestational diabetes mellitus (GDM). The poor response of insulin release, possible fat deposition in the skeletal muscle or ectopic fat deposition may cause dysfunctional homeostasis in GDM. This will definitely influence the fine tuning of metabolic machinery of a growing fetus. Children born with such subtle metabolic state will probably be more prone to glucose intolerance and ectopic lipid deposition. The finding that children born to GDM mothers are prone to glucose intolerance may be an eye-opener to monitor such children for beta cell function.
\end{abstract}

Keywords: glucose tolerance, oral glucose tolerance test, insulin resistance, gestational diabetes mellitus

Abbreviations: IR: insulin resistance; OGTT: oral glucose tolerance test; GDM: gestational diabetes mellitus

\section{Introduction}

Gestational diabetes mellitus (GDM) is one of the growing global health problems that afflicts the majority of pregnant population. It has been considered as one of the risk factors for developing diabetes in children born to such mothers $[1,2]$. GDM is reported to vary from $5.4 \%$ in Europe to $14.0 \%$ in Africa [3, 4]. In Asia, the prevalence of GDM is found to range from $0.7 \%$ to $51.0 \%$ [5-7]. Such wide variation cited in the literature may be due to variation in the screening method or diagnostic criteria or ethnicity or the population taken for the study. Yet GDM poses a great challenge for reproductive medicine and necessitates introspection and review the present status in the field of diabetes and reproductive health.

\section{Gestational diabetes mellitus and classification of diabetes mellitus}

Pregnancy is considered as a test for beta cell function and insulin reserve [2]. The classification of diabetes mellitus includes type 1 diabetes mellitus (T1DM) and type 2 diabetes mellitus (T2DM) as major categories apart from other 
glucose intolerant states. GDM is considered as a glucose intolerant state based upon hyperglycemia and perinatal outcomes [2].

\section{Diabetes can be classified into the following general categories:}

- Type 1 diabetes (due to autoimmune B-cell destruction, usually leading to absolute insulin deficiency).

- Type 2 diabetes (due to a progressive loss of B-cell insulin secretion frequently on the background of insulin resistance).

- Gestational diabetes mellitus (diabetes diagnosed in the second or third trimester of pregnancy that was not clearly overt diabetes prior to gestation).

- Specific types of diabetes due to other causes, e.g., monogenic diabetes syndromes (such as neonatal diabetes and maturity-onset diabetes of the young [MODY]), diseases of the exocrine pancreas (such as cystic fibrosis and pancreatitis), and drug or chemical-induced diabetes (such as glucocorticoid used in the treatment of HIV/AIDS or after organ transplantation) [2].

\section{Insulin and placental hormones in the early stage of pregnancy}

One needs to appreciate that womb is the most wonderful biochemical and metabolic chamber that primes and promotes the development, differentiation, and growth of a healthy fetus. It provides the required nutrition and nurture the fetus [3]. Maternal liver and adipose tissue initially become a storehouse of glycogen and triglycerides with the help of anabolic hormone insulin.

In other words, insulin sensitivity appears to be more at this stage where fuels are stored. With the growing fetus inside the womb, there is a surge of placental hormones; placental lactogen (hPL), placental growth hormone, progesterone, cortisol to overcome insulin action [4-8].

\section{- Insulin resistance}

This is achieved by developing an insulin resistant state which numbs the anabolic action of insulin promoting the release of glucose and fatty acids by enhancing glycogen breakdown and lipolysis in the liver and adipose tissue respectively [4-8] (Figure 1).

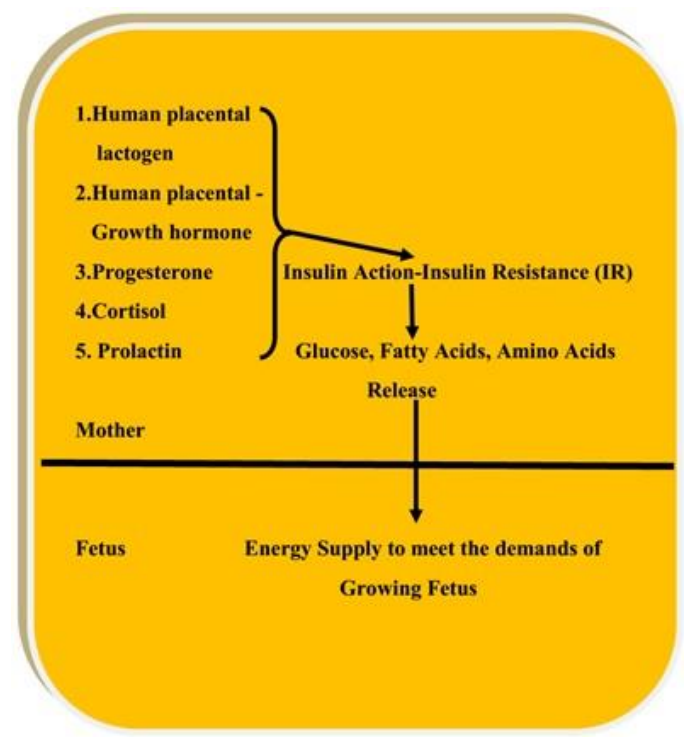

Figure 1: Insulin resistance in the pregnant mother to supply fuels to the growing fetus. 


\section{- Tumor necrosis factor alpha and insulin resistance}

Pregnancy is also considered as a pro-inflammatory state which releases factors like tumor necrosis factor alpha $(\mathrm{TNF} \alpha)$ which is reported to inhibit the binding of insulin to insulin receptor and therefore cause IR [5-9] (Figure 2).

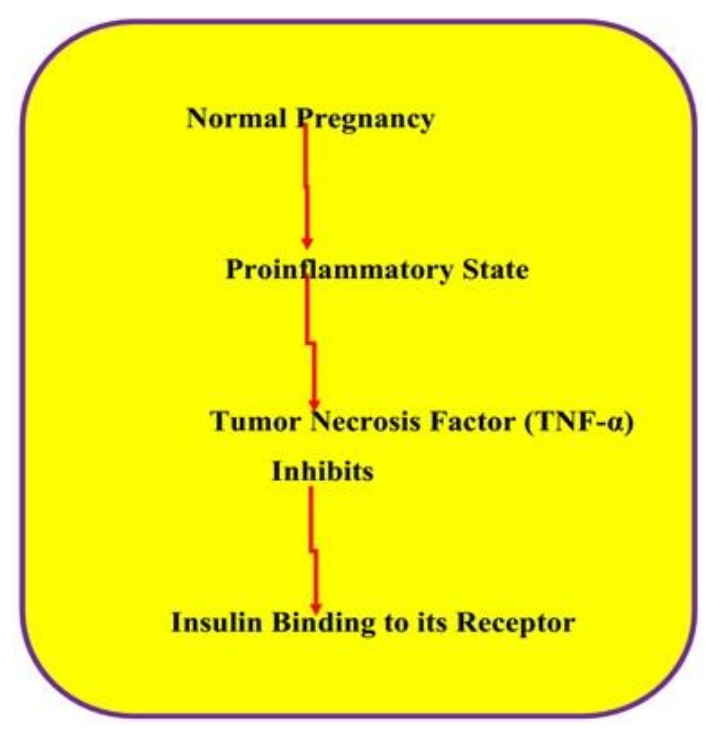

Figure 2: Possible role of tumor necrosis factor alpha in inducing insulin resistant state.

\section{- Normal insulin action}

Normally insulin binds to its receptor which has an intrinsic tyrosine kinase activity. The increased tyrosine kinase activity phosphorylates insulin receptor substrate (IRS-I). Phosphorylation of IRS-I receptor activates phosphatidylinositol-3-kinase activity which promotes the conversion of phosphatidylinositol bisphosphate $\left(\mathrm{PIP}_{2}\right)$ into phosphatidylinositol trisphosphate $\left(\mathrm{PIP}_{3}\right)\left(\mathrm{PIP}_{2} \rightarrow \mathrm{PIP}_{3}\right)[6-10]$.

Insulin translates its intracellular pleiotropic effects via $\mathrm{PIP}_{3}$ which acts through AKT-induced signaling promoting glycogen and triglyceride storage and also through the mTOR pathway promoting DNA synthesis, transcription, and translation inducing the synthesis of enzymes involved in glycogen storage and triglyceride formation [10].

\section{Placental hormones, progesterone and insulin resistance}

Increasing levels of maternal progesterone decrease PI3 kinase activity, thereby decreasing its insulin action. Elevated concentration of progesterone inhibits translocation of GLUT-4 receptors inhibiting glucose uptake and utilization by maternal adipose tissue and skeletal muscles respectively. It is shown that by experimental studies hPL can promote lipolysis and free fatty acid release into the fetus $[11,12]$. The cumulative actions of counter-regulatory hormones blunts insulin action promoting the release and availability of maternal fuels for the fetus to utilize.

\section{Gestational diabetes mellitus and glycemic control}

In a normal pregnancy, there is a steady fall in insulin levels from 18 to 28 weeks of gestation. After delivery, it returns to pre-pregnancy levels [13].

In GDM, the increase in basal insulin level is 3 or 4 times more than that of the levels seen in normal pregnancy. The hyperglycemic status continues as evidenced by oral glucose tolerance test (OGTT). The glucose intolerant state of GDM requires strict glycemic control and monitoring of fetal and maternal well-being. Increased BMI, advanced age, family history and, polycystic ovarian disease are considered to be the risk factors for GDM just like type 2 diabetes mellitus (T2DM) [9]. 


\section{Ectopic deposition of fat and insulin resistance}

There is a considerable evidence to suggest that ectopic deposition of fat in the pancreas, skeletal muscle and liver could be one of the factors that cause IR. Magnetic resonance study (MRS) has indicated that such deposition provides evidence to suggest that it is not just a dysfunctional metabolism of glucose but also lipid metabolism could be the cause for IR even in GDM [13].

\section{Diet and life style}

It is known that the western diet and sedentary lifestyle leads to fatty liver and intra-myocellular deposition of fat. This may lead to insulin resistance due to the possibility of substrates, glucose and fatty acids fighting for insulin receptor resulting in IR state in GDM like that may happen in obesity-related disorders. The increase in triglycerides in islet cells of the pancreas may blunt the response to glucose-induced release and synthesis of insulin [14-16]. This may lead to IR as depicted in the figure (Figure 3).

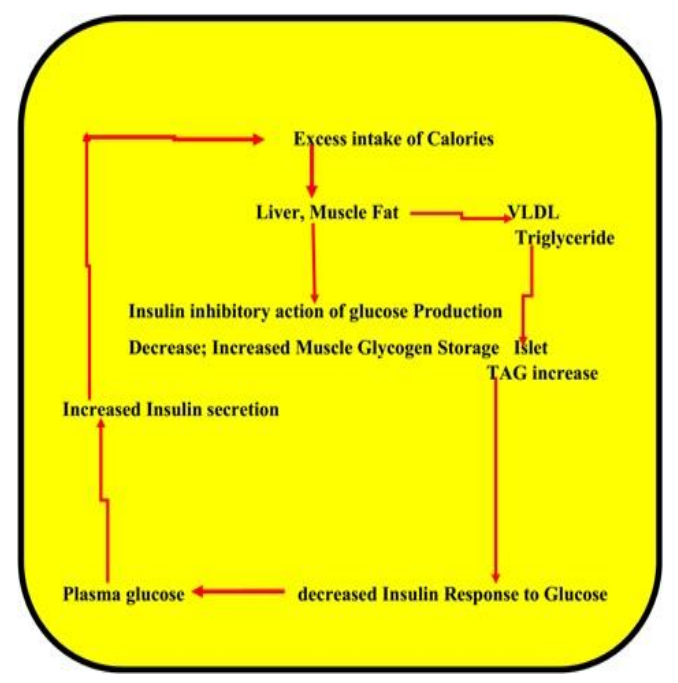

Figure 3: Excess calories and insulin resistance.

\section{GDM and Future Studies}

GDM is becoming one of the common gynecological problems faced by reproductive medicine. A pregnant mother with a previous history of GDM may be prone to incident T2DM. Children born to such mothers may also be susceptible to glucose intolerance and T2DM [1].

It is known that GDM may be a risk factor for preeclampsia and cardiovascular disease. The present trend that GDM may predispose the pregnant mothers and children to T2DM poses a challenge as well as lays emphasis on further research in understanding the biochemistry of GDM [2].

Overweight or obesity associated with any of the following factors including GDM during gestation, previous history of diabetes, ethnicity, hypertension, polycystic ovarian disease are cited to be the risk factors for precipitating diabetes for asymptomatic children [2].

\section{Conclusion}

GDM is a manifestation of pregnancy-related hyperglycemia due to a possible defect in up-regulation of insulin production to overcome IR or normalize insulin sensitivity to glucose intolerance. GDM is a sociological concern and creates a marital fear among families with challenging marital relationship [17]. Like diabetes, GDM is also a global economic issue and may be one of the health concerns of reproductive medicine. 


\section{References}

1. Blotsky AL, Rahme E, Dahhou M, et al. Gestational diabetes associated with incident diabetes in childhood and youth: a retrospective cohort study. CMAJ. 2019;191(15):e410-e417.

2. American Diabetes Association. Classification and diagnosis of diabetes: standards of medical care in diabetes-2018. Diabetes Care. 2018; 41(Suppl.1):S13-27.

3. Eades CE, Cameron DM, Evans JM. Prevalence of gestational diabetes mellitus in Europe: a meta-analysis. Diabetes Res Clin Pract. 2017;129:173-81.

4. Mwanri AW, Kinabo J, Ramaiya K, et al. Gestational diabetes mellitus in sub-Saharan Africa: systematic review and meta-regression on prevalence and risk factors. Tropical Med Int Health. 2015;20(8):983-1002.

5. Alfadhli EM, Osman EN, Basri TH, et al. Gestational diabetes among Saudi women: prevalence, risk factors and pregnancy outcomes. Ann Saudi Med. 2015;35(3):222-30.

6. Nguyen CL, Pham NM, Binns CW, et al. Prevalence of gestational diabetes mellitus in eastern and southeastern Asia: A systematic review and meta-analysis. J Diabetes Res. 2018;2018:1-10.

7. Wahi P, Dogra V, Jandial K, et al. Prevalence of gestational diabetes mellitus (GDM) and its outcomes in Jammu region. J Assoc Physicians India. 2011;59(4):227-30.

8. Sheriff DS. Medical Biochemistry. New Delhi: Jaypee Medical Publishers; 2004.

9. Barbour L, McCurdy C, Hernandez T, et al. Cellular mechanisms for insulin resistance in normal pregnancy and gestational diabetes. Diabetes Care. 2007; 30(2): 112-19.

10. Kirwan JP, Hauguel-De Mouzon S, Lepercq J, et al. TNF-alpha is a predictor of insulin resistance in human pregnancy. Diabetes. 2002; 51(7):2207-13.

11. Petersen MC, Shulman GI. Mechanisms of insulin action and insulin resistance. Physiol Rev. 2018;98(4):2133-2223.

12. Wada T, Hori S, Sugiyama M, et al. Progesterone inhibits glucose uptake by affecting diverse steps of insulin signaling in 3T3-L1 adipocytes. Am J Physiol Endocrinol Metab. 2010;298(4):e881-88.

13. González C, Alonso A, Alvarez N, et al. Role of 17 $\beta$-estradiol and/or progesterone on insulin sensitivity in the rat: implications during pregnancy. J Endocrinol. 2000;166(2):283-91.

14. Gloria T, Stephanie M, Miguel A, et al. Prevalence and risk factors of gestational diabetes mellitus: findings from a universal screening feasibility program in Lima, Peru. BMC Pregnancy Childbirth. 2018;18:303.

15. Tushuizen ME, Bunck MC, Pouwwels PJ, et al. Pancreatic fat content and beta-cell function in men with and without type 2 diabetes mellitus. Diabetes Care. 2007;30(11):2916-21.

16. Kautzky-Willer A, Krasak M, Winzer C, et al. Intramyocellular lipid concentration identifies impaired glucose metabolism in women with previous gestational diabetes. Diabetes. 2003;52(2):244-51.

17. Sheriff DS. Is it necessary to rename gestational diabetes mellitus? Indian J Endocrinol Metab. 2014;18(3):428. 